\title{
LA PROFESIONALIZACIÓN DE LOS ESTUDIOS DEL TRABAJO EN AMÉRICA LATINA RESULTADOS COMPARADOS DE UNA ENCUESTA REALIZADA EN DOS CONGRESOS DE ALAST
}

\author{
Jorge Walter, Diego Szlechter y Juan David Vanegas Daza
}

\section{Resumen}

La comunidad multidisciplinaria de los estudios del trabajo y las asociaciones nacionales y la regional que nuclean a los especialistas han tenido gran dinamismo en Latinoamérica durante las últimas décadas, en forma concomitante con un proceso de especialización de las ciencias sociales en general (De Sierra, Garretón, Murmis y Trindade, 2007). Este artículo compara los resultados de sendas encuestas respondidas voluntariamente por los miembros de la Asociación Latinoamericana de Estudios del Trabajo (ALAST) presentes en el sexto congreso, realizado en 2010 en México, y en el octavo, realizado en Buenos Aires en 2016. La comparación arrojó nuevos indicios de un "proceso de profesionalización" (Gaillard, Krishna y Waast, 1997) de los estudios del trabajo en la región.

Palabras clave: Profesionalización / comunidad científica / estudios del trabajo / América Latina.

\section{Abstract}

The professionalization of labour studies in Latin America: comparative results from a survey held in two ALAST congresses

The multidisciplinary community of labour studies and the national and regional associations that gather its specialists have been very dynamic in Latin America during the last decades, in a concomitant way with a specialization process of Social Sciences in general (De Sierra, Garretón, Murmis \& Trindade, 2007). This article compares the results of two surveys voluntarily answered by members of the Latin American Association of Labour Studies (ALAST) present at the Sixth Congress held in 2010 in Mexico and the Eighth Congress held in Buenos Aires in 2016. The comparison yielded new indications of a "professionalization process" (Gaillard, Krishna \& Waast, 1997) of labour studies in the region.

Keywords: Professionalization / scientific community / labour studies / Latin America.

Jorge Walter: Doctor en Sociología del Trabajo por la Universidad de París 3. Investigador principal del Consejo Nacional de Investigaciones Científicas y Técnicas (CONICET), sede Universidad de San Andrés, Argentina. Profesor titular regular de la Universidad de Buenos Aires (UBA), Argentina. E-mail: walter@udesa.edu.ar

Diego Szlechter: Doctor en Ciencias Sociales por la Universidad Nacional de General Sarmiento, Argentina. Investigador docente del Instituto de Industria de dicha universidad. Investigador del Consejo Nacional de Investigaciones Científicas y Técnicas (CONICET), Argentina. E-mail: diego_szlechter@yahoo.com.ar

Juan David Vanegas Daza: Sociólogo. Doctorando en Ciencias Sociales por la Universidad Nacional de General Sarmiento, Argentina. Becario doctoral del Consejo Nacional de Investigaciones Científicas y Técnicas (CONICET). Argentina. E-mail: juandavidvanegasdaza@gmail.com 


\section{Introducción ${ }^{1}$}

En este trabajo se examinan la formación, la inserción en instituciones, las fuentes de financiamiento de proyectos y la participación en redes locales e internacionales de los miembros de la Asociación Latinoamericana de Estudios del Trabajo (ALAST) que respondieron a tal efecto la segunda edición de una encuesta realizada durante el octavo congreso organizado por la institución en Buenos Aires en agosto de $2016^{2}$.

Los resultados de la primera encuesta realizada en el sexto congreso (México, 2010) dieron origen a una serie de publicaciones (Walter y Szlechter, 2012, 2014a, 2014b), en las cuales volcamos los resultados de la encuesta, que completamos con una serie de entrevistas individuales y colectivas a miembros de diferentes generaciones de la asociación.

Retomaremos aquí la problemática de la profesionalización planteada en dichas publicaciones iniciales, inspirada en los resultados de las investigaciones de nuestra contraparte europea en este proyecto (Gaillard, Krishna y Waast, 1997). Según dichos autores, la profesionalización es corolario de la institucionalización de una comunidad científica a escala nacional, cuando esta se ha consolidado y ya no se restringe al ámbito local. Esto último tiene lugar como consecuencia de la creación de instituciones educativas de nivel superior y estructuras específicas de formación, asociaciones profesionales y revistas, sistemas de referato para el financiamiento de proyectos, así como del establecimiento de patrones de calidad y productividad.

En el tránsito desde las comunidades científicas a los grupos profesionalizados, el carisma de los pioneros subsiste, pero en el ámbito simbólico, "rutinizado". Para que tenga lugar dicha transición es necesario que la comunidad se vincule con otros intereses por fuera de la disciplina, aproveche oportunidades para crear una demanda social y conforme a un sistema de reproducción de dicha demanda sostenible en el tiempo. Por otra parte, un grupo científico altamente profesionalizado no se restringe al ámbito nacio-

1 Este artículo analiza los resultados de una encuesta que tuvo su origen en un proyecto más amplio que contó con el apoyo del Programa EULAKS de la Unión Europea (Connecting Socioeconomic Research on the Dynamics of the Knowledge Society in the European Union and Latin American and Caribbean Countries).

Agradecemos las valiosas críticas realizadas por los evaluadores anónimos del artículo. 
nal y contribuye en alguna medida al avance del conocimiento a escala internacional. Posee además potencialidad en cuanto a establecer nexos viables con segmentos de la economía orientados hacia la producción.

Para dar cuenta de los procesos de institucionalización que conducen a la profesionalización, diseñamos la encuesta sobre la base de tres ejes analíticos clásicos: jerarquías, mercados y redes (Thompson, Frances, Levačić y Mitchell, 1991). El primero se refiere a las instituciones donde se desenvuelven los miembros de la asociación, las posiciones que ocupan y el tipo de tareas que realizan en ellas; el segundo, a las fuentes de financiación y el tipo de proyectos en los cuales están involucrados; el tercero está relacionado con las asociaciones a las que adhieren y las revistas científicas en las cuales someten su producción al juicio de los pares. Consideramos que estos ejes son complementarios y mutuamente necesarios para que el proceso de institucionalización tenga lugar.

En el análisis de los resultados de la encuesta realizada en 2010, nos preguntábamos en qué punto se ubicaban los miembros de la asociación asistentes al congreso que respondieron la encuesta - una muestra voluntaria de la comunidad subregional de estudiosos del trabajo- en el continuo que va desde la institucionalización a la profesionalización. ¿Reencontramos los indicios de esas mismas tendencias seis años después, en el extremo geográfico opuesto de América Latina?

\section{- La encuesta}

La primera encuesta llevada a cabo en el sexto congreso realizado en México en el año 2010 contó con financiamiento del Programa EULAKS de la Unión Europea, titulado Connecting Socio-economic Research on the Dynamics of the Knowledge Society in the European Union and Latin American and Caribbean Countries. Tanto en el diseño del cuestionario para la primera encuesta (en el cual introdujimos varias preguntas procedentes de la encuesta EULAKS) como en el análisis de la información recogida, contamos con la colaboración del equipo del Programa ${ }^{3}$. La sede argentina del proyecto fue la Universidad Nacional de General Sarmiento, que puso a su disposición a un becario de investigación como responsable de la carga y el procesamiento de los datos y la preparación de los cuadros. La segunda encuesta, realizada en el octavo congreso, en 2016 en Argentina, fue financiada con fondos de

3 Agradecemos especialmente la permanente colaboración de Rigas Arvanitis, investigador del Institut de Recherches pour le Développement (IRD, Francia), sede del proyecto, quien colaboró con nosotros en el diseño del formulario de la encuesta y en su realización durante el Congreso de ALAST en México, y aportó luego valiosas sugerencias para el procesamiento y el análisis de la información, así como numerosos comentarios que tuvimos en cuenta en el análisis de sus resultados. 
los investigadores argentinos que tomaron a su cargo el primer proyecto, integrantes de la Comisión Directiva de la Asociación ${ }^{4}$.

\section{Composición de los asistentes y el grupo encuestado ${ }^{5}$}

De acuerdo con las fichas de inscripción, al congreso realizado en Argentina asistieron 630 personas, a comparar con las 520 que participaron en el congreso mexicano. Confirmamos en primer lugar que la distancia tiene una fuerte influencia en la asistencia a los congresos. Un 47,9\% de los asistentes al congreso realizado en México eran mexicanos, porcentaje que se redujo al $8,1 \%$ en el de Buenos Aires. Inversamente, al congreso mexicano asistió un 14,8\% de argentinos, y al congreso de Buenos Aires un 44,3\%. Llama la atención sin embargo el gran número de argentinos que se trasladó a México en 2010, que supera ampliamente al de mexicanos que vinieron a Buenos Aires. Ello posiblemente se vincule con la mayor disponibilidad de financiamiento existente en 2010 en Argentina.

Es interesante además la presencia en Argentina de asistentes provenientes de cuatro nuevos países, dos de ellos más cercanos (Perú y Bolivia) y los otros dos europeos (Alemania e Italia). Francia y España continuaron presentes en Argentina, pero con un número de asistentes significativamente menor que en México. También concurrió al congreso realizado en Argentina una nutrida delegación uruguaya (también al congreso mexicano) y, sobre todo, de chilenos y colombianos (en Chile y Colombia se realizaron los dos precongresos ALAST anteriores al congreso de Buenos Aires). Colombia, por su parte, se postuló y fue elegida como la futura sede de la Asociación para el período 2016-2019.

La encuesta fue respondida voluntariamente por el $25 \%$ de los asistentes (158 personas), una proporción superior a la lograda en el congreso mexicano $(23,6 \%, 116$ personas). Dichas tasas resultan insuficientes a los efectos de establecer comparaciones entre países, salvo los más grandes (Brasil, México y Argentina). A lo largo del artículo nos referiremos por lo tanto a indicios que surgen del análisis de la información, dadas las fuertes limitaciones que tiene nuestra fuente de información ${ }^{6}$.

4 Jorge Walter presidió la ALAST durante el período 2013-2016 y Diego Szlechter se desempeñó como secretario en el mismo período. El asistente a cargo del procesamiento de los datos de la encuesta realizada en Argentina fue Juan David Vanegas Daza, becario doctoral del CONICET con sede en el Instituto de Industria de la Universidad Nacional de General Sarmiento.

5 Por motivos prácticos vinculados con la edición hemos debido restringir al máximo el uso de cuadros y gráficos para representar la información.

6 Típicamente, por cuestiones de acceso a fondos de financiación, hay una proporción dominante de investigadores formados provenientes de países lejanos (por ejemplo, argentinos en México, mexicanos en Argentina) y una fuerte presencia de estudiantes e investigadores más jóvenes del país donde se realiza el congreso o de países vecinos. 
La tasa de respuesta de los países más grandes fue dispar. Mientras respondió un $37 \%$ de los mexicanos que asistieron al congreso, lo hizo un 31\% de los brasileros, manteniendo ambos países los altos índices de respuesta del congreso de México. Sorprende sin embargo la baja tasa de respuesta de los argentinos (16,5\%), muy inferior al promedio, menor incluso al guarismo alcanzado en el congreso de México (22,1\%).

Los datos recogidos en el congreso de Buenos Aires nos permitieron distinguir a los investigadores (con título de maestría o superior) y a los estudiantes (de licenciatura) que respondieron la encuesta. Un 24\% de las respuestas (un 43,5\% en el caso de los argentinos) provino de estudiantes, es decir, de personas sin publicaciones ni trayectoria en la disciplina.

\section{- Edades}

La composición por edades del grupo encuestado en Argentina tuvo una distribución más equilibrada que en México entre jóvenes estudiantes y adultos al final de su carrera. En Argentina la forma graficada es claramente piramidal a partir de los 40-50 años, siendo esta categoría intermedia la dominante $(28,5 \%)$, y se reduce levemente hacia las categorías de menor edad $(25,1 \%$ entre 30 y 40 años y $14,5 \%$ entre 20 y 30 años), un indicio de la existencia de bases sólidas para el desarrollo y la renovación de la institución.

\section{- Estudios}

La mayoría de los encuestados en Argentina (76\%) posee diplomas de posgrados de investigación (en México, el 84,6\%). El 48,1\% posee un doctorado (en México, el 49\%) y un posdoctorado (el 3,8\%), indicios reiterados de una fuerte orientación hacia la investigación por parte de los miembros de la Asociación.

A diferencia de México, donde el grupo de 50-60 años tenía el mayor número de doctorados, en Argentina es la franja etaria 40-50 la que detenta ese podio. Respecto a las maestrías, si bien tanto en Argentina como en México el grupo más numeroso es el de 30-40 años, la proporción de este rango etario se reduce a la mitad en el Congreso en Argentina respecto del de México. Si la encuesta de México daba cuenta de una notoria propensión a iniciar y culminar tardíamente los estudios de posgrado, la llevada a cabo en Argentina es testigo de un adelantamiento de ese proceso - especialmente en los doctorados - , tendencia claramente relacionada con la institucionalización de los estudios del trabajo en la región.

Otro dato interesante que surge de la encuesta realizada en Argentina tiene que ver con la mayor proporción de personas con doctorados entre los mexicanos y con maestrías y posdoctorados entre los brasileños. Los argentinos se sitúan en un punto intermedio entre dichos países en los tres niveles 
de formación. Lo anterior está posiblemente asociado con el grado de institucionalización de las formaciones específicas de alto nivel en esos países.

En cuanto a la especialidad de los diplomas, la Sociología - disciplina fundadora de la Asociación - continúa siendo dominante. Si sumamos a ella los diplomas en Ciencias Sociales y Estudios Laborales, se alcanza el 45,6\% de los encuestados. El resto de las disciplinas presentes (por ejemplo, Psicología y Educación) suman un 19\%, confirmando un rasgo muy valorado por la Asociación: su apertura al debate interdisciplinario.

\section{Inserción institucional}

Nos referiremos a continuación al tipo de institución donde el encuestado realiza tareas de investigación, al tipo de contrato que lo vincula con ella y a la distribución en el uso de su tiempo entre la docencia, la investigación, la responsabilidad institucional y la consultoría.

$\mathrm{Al}$ igual que en México, prácticamente la totalidad de los encuestados afirma desarrollar actividades de investigación, cuyos productos estaban presentando en el congreso. Más adelante nos referiremos a la proporción del tiempo de trabajo que dedican a esa actividad en las instituciones donde se desempeñan.

Un 76,5\% de los encuestados en México realizaba actividades de investigación en una universidad. En Argentina, esa proporción fue casi exactamente la misma $(76,8 \%)$, lo cual confirma la importancia de las universidades como lugar de la investigación en América Latina.

\section{- Tipos de contrato}

Un 83,3\% de los encuestados en Argentina contaba con contratos de trabajo de tiempo completo, ya fuesen permanentes $(65,2 \%)$ o transitorios $(18,1 \%)$, cifras ligeramente superiores a las registradas en México.

\section{- Tipos de contrato y edad}

Si se cruzan el tipo de contrato y el rango de edad, en el grupo encuestado en Argentina se observa que los más jóvenes tienen una baja proporción relativa de contratos permanentes y una alta proporción relativa de contratos temporales y de tiempo parcial, lo cual confirma la forma clásica de selección para el reclutamiento en puestos de investigación.

\section{- Contrato, cargo y edad}

La proporción de encuestados que ocupaba cargos de responsabilidad en sus instituciones de pertenencia fue elevada y similar entre el sexto congreso $(38,2 \%)$ y el octavo $(37,7 \%)$, además de involucrar a una parte sustancial de 
las personas con contratos de carácter permanente $(57,8 \%$ de los encuestados en 2010 y $63,9 \%$ en 2016).

Asimismo, la ocupación de cargos institucionales crece con la edad, con un máximo en el rango etario entre los 40 y 50 años, y extendiéndose hasta la edad de la jubilación.

El alto porcentaje que arroja la encuesta en materia de cargos de responsabilidad es un indicio reiterado del sólido posicionamiento institucional de los científicos del trabajo de la región.

\section{- País y tipos de contrato}

Es interesante por último constatar entre los mexicanos que respondieron la encuesta en Argentina la ausencia de personas sin contratos de tiempo completo (que seguramente no cuentan con financiamiento específico para concurrir a congresos), así como la mayor proporción de brasileros que de argentinos con contratos temporales de tiempo completo, y la relación inversa en cuanto a personas con contratos de tiempo parcial.

\section{Uso del tiempo de trabajo}

En cuanto a la distribución del uso del tiempo de trabajo entre la investigación, la docencia y la consultoría/servicios técnicos, se mantienen las mismas prevalencias registradas en ambas encuestas. La investigación predomina ampliamente en las categorías de dedicación superiores al 50\% del tiempo de trabajo, confirmando la orientación de los miembros de la asociación hacia la investigación. La docencia prima en segundo lugar en las categorías entre $26 \%$ y $75 \%$ del tiempo de trabajo, y la gestión en las categorías entre $1 \%$ y $50 \%$ del uso del tiempo. Por último, la consultoría y la asistencia técnica son ampliamente dominantes en la categoría $0 \%$ de dedicación.

Sin embargo, constatamos algunas diferencias significativas: un aumento relevante del tiempo destinado a la docencia en las categorías intermedias de dedicación, y a la gestión y la consultoría en las categorías de más baja dedicación. ¿Presiones crecientes de la docencia sobre la investigación en las universidades?, ¿estímulos crecientes del financiamiento en favor del vínculo entre la investigación y la actividad productiva? ${ }^{7}$, ¿sesgo Cono Sur en la composición de los encuestados?

\section{Redes profesionales y científicas}

$7 \quad$ Coincidimos sin embargo con el punto de vista de Leonardo Vaccarezza en cuanto a que "No parece ser la cuestión del conocimiento como estrategia principal lo que nos permitiría describir la agencia de los investigadores, sino los distintos componentes relacionales de la vida académica que les permite mantenerse e identificarse (reproducirse) como profesionales académicos" (Vaccarezza, 2000, p. 42). 
En este punto nos referiremos al lugar donde los encuestados realizaron estudios superiores, a sus estadías en el extranjero, a su cooperación con colegas extranjeros, a los temas en los cuales se han especializado y a los motivos que tuvieron para elegirlos, a su pertenencia a asociaciones, a su participación en la creación de revistas y en comités editoriales, a las revistas en las cuales publican y a su participación en congresos.

\section{- Estudios y estadías en el extranjero}

Nos referiremos en primer lugar al sitio de realización de los estudios superiores, a la vez como espacios de oportunidad para el tejido de redes locales e internacionales y como indicio de institucionalización de los estudios del trabajo en la región.

En la encuesta realizada en 2010 en México, el 42,2\% de los encuestados había realizado estudios superiores en el extranjero. En el octavo congreso, realizado en 2016 en Argentina, esa proporción reunió solo a un tercio de los encuestados $(33,5 \%)$. Si analizamos la distribución por categorías de edad en el congreso argentino, vemos que el $71 \%$ de los mayores de 60 años (ese porcentaje es superior al doble del promedio general) realizó estudios en el exterior, posiblemente por la falta de oportunidades locales de formación en el momento en el que realizaron sus estudios, y menos de la mitad (14\%) de los menores de 30 años - quizás por el motivo inverso- hizo lo propio. Si se comparan esos datos con los del congreso mexicano, llama la atención que el porcentaje de la franja de 20 a 30 años casi triplica la proporción de diplomados en el extranjero registrada en el congreso argentino.

\section{Idioma de estudios}

En cuanto a la lengua de los países donde los encuestados realizaron sus estudios superiores, la comparación entre el sexto y el octavo congreso da cuenta de una reducción de la influencia de los países de lengua española y francesa, y un incremento de la influencia de aquellos de lengua inglesa y portuguesa, esto último debido probablemente a que el último congreso se realizó cerca de Brasil y congregó a una importante cantidad de personas de ese país.

Tal como sostuvimos en un artículo anterior (Walter y Szlechter, 2014b, p. 221), los estudios de posgrado realizados en el extranjero tienen un peso importante y probablemente una gran influencia sobre el desarrollo de la disciplina en la región ${ }^{8}$. No obstante, la mayor y creciente parte de los posgrados

8 Vinck constata, sin embargo, que la realización de un doctorado en el exterior no alcanza para establecer relaciones internacionales durables (Vinck, 2013). Los beneficios de este tipo de estadías dependen del intercambio efectivo de conocimientos y de su utilización. 
ha sido cursada localmente ${ }^{9}$, lo cual evidenciaría que la disciplina se ha instalado ya sólidamente en la región a partir de la creación de formaciones locales de posgrado orientadas hacia la investigación (maestrías y doctorados).

Un indicador de esto último son las estadías de investigación realizadas en el extranjero. Un 29,7\% (47 personas) de los encuestados en Buenos Aires (158) declaró haber realizado estadías de investigación en el extranjero, principalmente en Francia, Brasil e Inglaterra. Veamos ahora la percepción de los encuestados sobre el rol de la cooperación internacional en el desarrollo de sus trabajos.

\section{- Percepciones sobre la cooperación internacional}

Las respuestas a la pregunta sobre el valor atribuido por los encuestados a la cooperación con colegas del extranjero las ordenamos jerárquicamente, de mayor a menor, sumando a tal efecto las opciones: "lo considero esencial" y "lo considero muy importante".

Para comparar las respuestas del sexto y el octavo congresos tomamos como punto de referencia la jerarquía del sexto congreso y examinamos las similitudes y cambios de orden en relación con la jerarquía resultante de la encuesta realizada en el octavo congreso (Cuadro 1).

\section{Resultados de la cooperación internacional}

Sorprenden, ante todo, las fuertes similitudes entre ambas jerarquizaciones. A punto tal, y es este posiblemente el mayor interés de la comparación entre ambos congresos, que la estabilidad de las percepciones - no obstante el cambio en la composición de quienes respondieron la encuesta - nos permite esbozar una hipótesis sobre la existencia de una verdadera cultura compartida por los miembros de la asociación. ¿Qué rasgos tiene dicha cultura en lo atinente al rol de la cooperación?

En los primeros lugares de la jerarquía aparece el camino crítico que va desde la presentación de una ponencia en un congreso - y el desarrollo de vínculos con contrapartes extranjeras - a su publicación en una revista científica extranjera. Asociado con todo lo anterior viene el desarrollo de nuevas temáticas y métodos de investigación y la participación en nuevos proyectos científicos.

9 Este fenómeno es mencionado por Gaillard, Gaillard, Russell Barnard, Galina, Canesse, Pellegrini, Ugartemendia y Cardenas (2013, p. 183) para las ciencias naturales en el caso argentino: "Argentina es por lejos el país donde mayor proporción de doctorados es obtenida en el país (83\%). Los investigadores argentinos tienen un tipo de carrera más convencional que sus colegas latinoamericanos, quienes tienden a obtener su doctorado a mayor edad" (traducción nuestra). 
En los últimos cuatro lugares aparecen el reconocimiento de la calidad científica (de la propia producción y de la institución de pertenencia) y la obtención de financiamiento (y de otro tipo de recursos eventualmente escasos, como los datos y la información), que es la forma como se expresaría concretamente dicho reconocimiento.

En síntesis: un fuerte énfasis en la producción y en el valor del intercambio para la producción, y una menor atención al acceso a recursos y al logro de reconocimiento. ¿Significa esto último que la cooperación internacional no se valora en tanto fuente de recursos o que no se necesitan ni se intenta obtenerlos gracias a ella? La respuesta a otras preguntas nos permitirá avanzar en esta dirección.

Cuadro 1. Resultados por orden de importancia de la colaboración con colegas extranjeros [sexto y octavo congresos].

\begin{tabular}{lcc}
\hline \multirow{2}{*}{ Resultados de la colaboración con colegas extranjeros } & \multicolumn{2}{c}{ Orden de importancia } \\
\cline { 2 - 3 } & $\begin{array}{c}\text { Sexto } \\
\text { congreso }\end{array}$ & $\begin{array}{c}\text { Octavo } \\
\text { congreso }\end{array}$ \\
\hline Desarrollo de nueva temática de investigación & 1 & 5 \\
\hline Participación en congresos, formaciones, etcétera & 2 & 1 \\
\hline Publicación en revistas extranjeras & 3 & 2 \\
\hline Refuerzo de los vínculos con contrapartes internacionales & 3 & 4 \\
\hline Desarrollo de nuevas metodologías de investigación & 4 & 5 \\
\hline Participación en nuevos proyectos científicos & 5 & 3 \\
\hline Reconocimiento en la propia institución & 6 & 8 \\
\hline Acceso a datos e información no disponible en su país & 7 & 7 \\
\hline Reconocimiento científico a escala internacional & 8 & 6 \\
\hline Aumento del financiamiento para su institución & 9 & 9 \\
\hline
\end{tabular}

Fuente: Encuesta Los Estudios del Trabajo en América Latina (ver Anexo].

- Temas de los proyectos en curso

A los efectos de la comparación, nos limitamos a seleccionar los temas en los cuales se enmarcaban más proyectos sobre la base de una categorización ex post, a partir de los títulos de los proyectos en los cuales estaban involucrados los encuestados.

Hay fuertes coincidencias entre los temas, casi una década después del sex to congreso. Los problemas del mercado de trabajo y la cuestión sindical continúan a la cabeza. Se repiten el tema de las condiciones de trabajo, del 
riesgo y la salud en el trabajo, las temáticas del género, las cuestiones de juventud, educación y trayectorias laborales, y los problemas laborales vinculados con la migración. En el congreso mexicano los encuestados mencionaron frecuentemente una problemática - la economía del conocimiento y la innovación - que no se vuelve a mencionar con la misma fuerza en el octavo congreso. Temas tradicionales aparecen en uno $\mathrm{u}$ otro congreso, como el trabajo de los mayores, el trabajo en la economía social y en el medio rural, el trabajo y la subjetividad. En el octavo congreso se mencionan proyectos sobre los trabajos de cuidado y los estudios del management y la organización.

\section{Elección del tema de investigación}

En este apartado, los encuestados debían optar por una serie de razones para elegir el tema de investigación en el cual estaban trabajando. Los motivos de la elección fueron en ambos congresos los mismos, con muy ligeras variaciones. Nuevamente, se plantea aquí la pregunta sobre los rasgos que caracterizarían la cultura institucional de la Asociación (Cuadro 2).

Cuadro 2. Razones para elegir el tema de investigación, por orden de importancia.

\begin{tabular}{lcc}
\hline & Orden de importancia \\
\cline { 2 - 3 } Razones para la elección & $\begin{array}{c}\text { Sexto } \\
\text { congreso }\end{array}$ & $\begin{array}{c}\text { Octavo } \\
\text { congreso }\end{array}$ \\
\hline Interés personal y/o curiosidad intelectual & 1 & 2 \\
\hline Importancia social y política del tema para Latinoamérica & 2 & 1 \\
\hline Tema de su investigación para la maestría o el doctorado & 3 & 3 \\
\hline Tema novedoso al que se puede contribuir de manera original & 4 & 4 \\
\hline Tema de investigación de las redes científicas de pertenencia & 5 & 5 \\
\hline El tema corresponde a las orientaciones de su equipo de investigación & 6 & 6 \\
\hline Permite publicar en revistas y libros a escala internacional & 7 & 8 \\
\hline Tema vinculado con actividades docentes & 8 & 7 \\
\hline Tema con amplia disponibilidad de financiamiento & 9 & 11 \\
\hline Respuesta a un pedido de organismos nacionales o internacionales & 10 & 9 \\
\hline Respuesta a un pedido de sindicatos, ministerios u otros organismos nacionales & 11 & 10 \\
\hline
\end{tabular}

Fuente: Encuesta Los Estudios del Trabajo en América Latina (ver Anexo).

Entre las razones más importantes para elegir un tema de investigación, las principales coincidencias entre las respuestas recogidas en ambos congresos se refieren al fuerte énfasis en el "interés personal y/o la curiosidad intelec- 
tual" y en la "importancia social y política del tema para América Latina". Entre los motivos a los que se asigna menor importancia se encuentran la demanda "de organismos nacionales o internacionales" y "de sindicatos, ministerios u otros organismos nacionales" $\mathrm{y}$, coherentemente con ello, que se trate de un "tema con amplia disponibilidad de financiamiento".

Lo anterior puede interpretarse como una reivindicación de la autonomía personal frente a las prioridades establecidas por instituciones como los ministerios o los sindicatos al efectuar sus demandas.

Si se combinan estos resultados con los del Cuadro 1 (sobre la importancia de la colaboración con colegas del extranjero), en el cual se constataba la importancia fundamental de ese tipo de vínculos para la renovación teórica y metodológica de las investigaciones, se confirma la impresión sobre una cierta falta de autonomía de la producción local. Esta falta de autonomía se refleja en su escasa conexión con las demandas locales y en una fuerte referencia a las temáticas y los problemas tal como vienen definidos desde dentro del campo científico y de acuerdo con contextos ajenos a los del propio investigador. Cabe sin embargo preguntarnos, como lo hicimos al comentar este cuadro a partir de los resultados de la encuesta realizada en el sexto congreso, si las demandas gubernamentales o sindicales realmente existen e interpelan la producción de la disciplina y, cuando eso sucede ${ }^{10}$, en qué medida participaron los investigadores en la definición de los temas prioritarios de investigación.

Podemos reiterar, en consecuencia, que estas observaciones son un llamado de atención respecto a la necesidad de un mayor esfuerzo para que se cumpla la finalidad de la Asociación en cuanto a promover debates que respondan a la especificidad del propio contexto y a influir sobre los organismos públicos y las organizaciones sociales, eventualmente colaborando con ellos a precisar sus demandas.

\section{- Asociaciones y revistas}

Un 26,6\% de los encuestados en Argentina afirma haber participado en la creación y la dirección de instituciones ligadas a las problemáticas del trabajo. Entre las más mencionadas se encuentran la propia ALAST, la Asociación Mexicana de Estudios del Trabajo (AMET); la Asociación Brasileña de Estudios del Trabajo (ABET) y la Asociación Argentina de Especialistas en

10 Como sucedió por ejemplo con la Subsecretaría de Programación Técnica y Estudios Laborales del Ministerio de Trabajo, Empleo y Seguridad Social de Argentina que, bajo la dirección de una fundadora de la ALAST, promovió investigaciones y fue ámbito de publicación de numerosos estudios e investigaciones y, a partir de 2005, de la Revista Trabajo, disponible en línea: <http://www.trabajo.gob.ar/estadisticas/revista/>. 
Estudios del Trabajo (ASET), a cargo de la organización de los congresos nacionales y el latinoamericano de la especialidad.

Los encuestados afirman haber participado además en la creación de la Revista Latinoamericana de Estudios del Trabajo (RELET) de ALAST y la Revista Latinoamericana de Derecho Social, así como de las nacionales de la especialidad, como la Revista Estudios del Trabajo de ASET en Argentina, la Revista da ABET y la Revista de Ciências do Trabalho en Brasil, la Revista Trabajo en México, y la nueva revista colombiana en línea E-trabajo.

Gracias a este movimiento, los estudios del trabajo disponen en Latinoamérica de una gran variedad de espacios de debate sobre la producción regional de los especialistas, que abordan la temática desde diferentes disciplinas. En efecto, las asociaciones nacionales y también la ALAST (que conservó por memoria la "S" de Sociología en su sigla, pero la sustituyó en el texto por la palabra Estudios) han hecho suya la apertura en sus denominaciones institucionales.

- Razones para elegir una revista

Este punto procede de dos nuevas preguntas que agregamos en la encuesta realizada en el octavo congreso. La primera, referida a la importancia (entre 1 y 10, donde 10 es la máxima importancia) atribuida a diferentes razones para elegir una revista (referato, indización, factor de impacto, en ese orden); la segunda, referida a la importancia que les dan las instituciones donde se desenvuelven los investigadores a tales factores de las revistas en las que publicaron en la evaluación de su desempeño.

Referato, indización, factor de impacto

Para visualizar los resultados de la primera pregunta decidimos agrupar las categorías en tres segmentos (Gráfica 1): inferior (de 1 a 4), intermedio (entre 5 y 7) y superior (entre 8 y 10). Resulta sorprendente la importancia superior asignada al factor de impacto (que claramente distingue a las ciencias duras). 
Grafica 1. Entre las razones para desear publicar en una revista, ¿qué importancia le asigna al referato, la indización y el impacto? (octavo congreso).

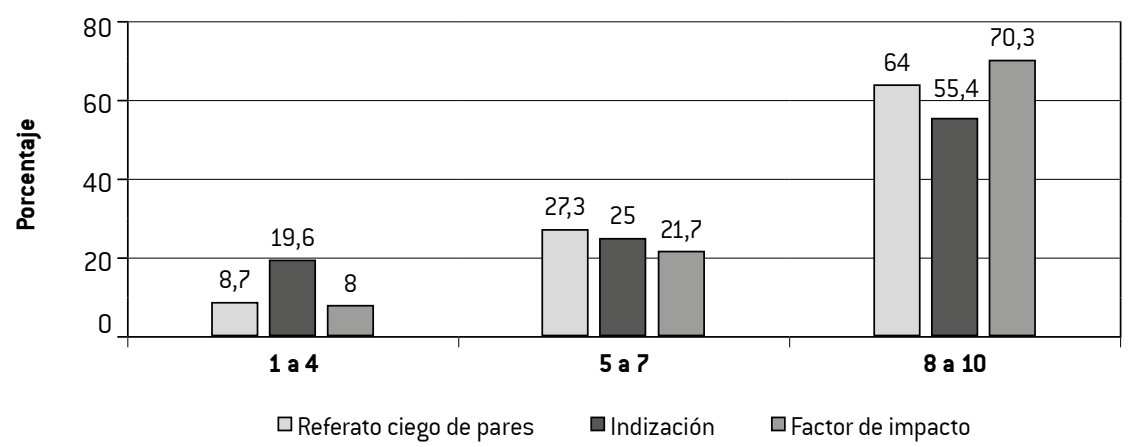

Fuente: Elaboración propia.

Influencia en la evaluación de desempeño

Las categorías "muy importante" y "esencial” agrupan en este caso el 81,3\% de las respuestas. La fuerte exigencia en la selección de las revistas en las que publicar se relaciona con la percepción de la gran importancia que asignan las instituciones de pertenencia a la calidad de las publicaciones en las evaluaciones de desempeño. No podemos afirmar, sin embargo, porque no lo hemos preguntado, que el "factor de impacto" sea un criterio determinante adoptado por ellas al jerarquizar las publicaciones de sus miembros.

\section{Fuentes de financiación}

A continuación examinaremos las fuentes de financiamiento según su origen nacional o internacional durante los cinco años previos a la realización de cada encuesta, y luego el tipo de proyectos financiados.

\section{- Nacionales versus internacionales}

Las encuestas realizadas en ambos congresos dan cuenta del mismo fenómeno. El financiamiento de los proyectos es de fuente básicamente nacional. Más de un $70 \%$ de ellos está financiado en más de un $80 \%$ por fondos de ese origen.

El financiamiento internacional, sin embargo, no es despreciable, pues tiene impacto significativo en categorías intermedias de financiación (por ejemplo, entre un $20 \%$ y un $40 \%$ de los fondos), como complemento de las fuentes locales. 


\section{- Según tipos de proyecto}

La información sobre la pregunta formulada en México sobre los tipos de proyecto se reveló difícil de procesar, razón por la cual debimos modificar la categorización y no podemos realizar una comparación. La nueva pregunta puso en evidencia una paridad entre los proyectos individuales (posiblemente vinculados con la preparación de tesis) y los colectivos con financiamiento nacional ( $44 \%$ y $43 \%$ de los proyectos respectivamente). Tienen un carácter cuantitativamente marginal $(12,9 \%)$ los proyectos colectivos e individuales con financiamiento internacional parcial (9\%), y los proyectos colectivos con financiamiento internacional total $(3,9 \%)$. No se mencionó ningún proyecto individual con financiación internacional.

\section{- Razones para no responder a convocatorias internacionales}

Visto el carácter relativamente marginal del financiamiento internacional de proyectos, formulamos en ambos congresos una pregunta relativa a las razones para no postular a ellos. El financiamiento local es sinónimo de autonomía con respecto a las agendas de investigación de otros países, pero la no presentación a convocatorias internacionales implica desaprovechar una fuente importante de recursos.

Observemos en primer lugar que la falta de interés no es el motivo de la no presentación a convocatorias (Cuadro 3). La forma negativa de la pregunta nos obliga a efectuar de entrada dicha constatación: la financiación internacional sería más que bienvenida, es la opinión sobre todo de los encuestados en el octavo congreso. En ambos congresos, las respuestas coinciden además, con ligeras variaciones, sobre las seis razones más importantes para no acceder a tales fuentes de financiación. Proponemos la siguiente interpretación: las dificultades provienen sobre todo de la falta de apoyo institucional en las universidades para: a) que los investigadores estén informados sobre las convocatorias, b) dispongan del tiempo y realicen el esfuerzo necesario para presentarse (habida cuenta de que los montos no siempre se correlacionan con el esfuerzo necesario para obtenerlos y administrarlos), c) apoyar técnicamente y capacitar a los investigadores sobre el fárrago de requisitos a cumplir y en la preparación de las presentaciones, c) colaborar con ellos en la administración de los proyectos, sin agregar burocracia propia a la burocracia internacional. En otras palabras: el posicionamiento institucional de los estudiosos del trabajo no significa que sus instituciones sean un campo propicio para el desarrollo de la investigación, al menos en materia de ayuda a la presentación en proyectos y a su administración, una habilidad más institucional que individual (Van Looy, Ranga, Callaert, Debackere y Zimmermann, 2004). 
Cuadro 3. Razones para no presentarse a las convocatorias internacionales.

\begin{tabular}{llc}
\hline Razones para no presentarse & $\begin{array}{c}\text { Sexto } \\
\text { congreso }\end{array}$ & $\begin{array}{c}\text { Octavo } \\
\text { congreso }\end{array}$ \\
\hline Falta de información sobre las convocatorias & 1 & 1 \\
\hline Falta de experiencia o capacitación para someter propuestas a las convocatorias & 2 & 2 \\
\hline Falta de tiempo & 3 & 3 \\
\hline Demasiada selectividad de las convocatorias & 4 & 6 \\
\hline Esfuerzo demasiado importante con relación al monto que se obtiene & 5 & 5 \\
\hline Dificultades vinculadas a las normativas financieras y contables de su institución & 6 & 4 \\
\hline No necesita este apoyo & 7 & 10 \\
\hline Problemas culturales y de idioma & 8 & 7 \\
\hline Su institución no tiene el nivel científico suficiente & 9 & 9 \\
\hline Falta de un marco legal o jurídico nacional que permita participar & 10 & 8 \\
\hline
\end{tabular}

Fuente: Encuesta Los Estudios del Trabajo en América Latina (ver Anexo).

\section{Síntesis y conclusión}

Como ya señalamos en anteriores publicaciones, la comunidad multidisciplinaria de los estudios del trabajo y las asociaciones nacionales y la regional que nuclean a sus miembros han tenido gran dinamismo en América Latina durante las últimas décadas, en el marco de un proceso de "diversificación" (De Sierra, Garretón, Murmis y Trindade, 2007, p. 46) o "especialización" (Murmis, 2007, p. 83) de las ciencias sociales, que tuvo lugar tras el retorno de las democracias en los años ochenta.

Los resultados de la encuesta realizada en 2010 en el congreso mexicano habían ofrecido indicios consistentes de un proceso avanzado de institucionalización en los tres ejes mencionados en la introducción (jerarquías, mercados y redes). ¿Hay indicios, en la nueva encuesta realizada en 2016 en el otro extremo del subcontinente, de una profundización o de un cambio en dichas tendencias?

Nos referiremos en forma sintética primero a las similitudes y luego a los cambios observados entre ambos congresos, y propondremos luego una conclusión de cierre.

En cuanto a las semejanzas, constatamos:

- El carácter dominante de la disciplina fundadora (la sociología), pero la presencia de una amplia variedad de otras disciplinas.

- La mayoría de los encuestados en Argentina (76\%) y en México (84,6\%) posee diplomas de posgrados orientados hacia la investigación. 
- El 76\% de los entrevistados en ambos congresos (la proporción es idéntica) se desempeña en una universidad.

- Entre un $76 \%$ (México) y un $82 \%$ (Argentina) lo hace con contratos o cargos de tiempo completo.

- Un 38\% de los entrevistados en ambos congresos ocupa posiciones de responsabilidad jerárquica, indicio reiterado de un sólido posicionamiento institucional.

- Se mantienen en ambos congresos las mismas prevalencias en cuanto al uso del tiempo:

- La investigación predomina en las categorías de uso del tiempo superiores al $80 \%$.

- La docencia en las intermedias (entre un $26 \%$ y un $75 \%$ de dedicación).

- La consultoría y la asistencia técnica prevalecen en la categoría $0 \%$ de dedicación.

- No obstante el cambio en su composición, los encuestados en ambos congresos tienen percepciones sorprendentemente similares sobre los resultados de la cooperación con colegas del extranjero, lo cual nos invita a preguntarnos sobre la existencia de una cultura institucional. Las coincidencias se refieren a:

- Las consecuencias positivas que se atribuyen a la cooperación con colegas extranjeros en lo que respecta a la renovación teórica y metodológica y la publicación en revistas internacionales.

- El comparativamente escaso beneficio que se obtiene de ella para el logro de reconocimiento local e internacional y la obtención de recursos (financiamiento, información).

- Se reiteran en ambos congresos los temas más investigados, que reúnen alrededor de un 10\% de los proyectos: trabajo precario (en su vínculo con el empleo, el desempleo y la tercerización) y sindicalismo son los más recurrentes.

- También nos sorprendieron las fuertes similitudes en la forma de jerarquizar los criterios de elección de los temas de investigación:

- Los criterios fundamentales son, en primer lugar, el interés y la curiosidad personales y, en segundo lugar, la importancia social y política del tema para América Latina.

- No se le atribuye gran influencia a la disponibilidad de financiamiento ni a las demandas de instituciones como los organismos internacionales, el Estado o los sindicatos. 
- Otra fuerte coincidencia refiere a las fuentes de financiación: más de un $70 \%$ de los proyectos (74,7\% en México, 76,7\% en Argentina) ha sido financiado en una proporción superior al $80 \%$ mediante fondos de origen nacional.

- La mitad de esos proyectos es de carácter colectivo.

- La otra mitad es individual (posiblemente en su mayoría de tesistas e investigadores al comienzo de sus carreras).

- El financiamiento internacional no es despreciable, pero tiene sobre todo impacto en las categorías intermedias de financiación, menores al $80 \%$ y superiores al $20 \%$ del monto total de los proyectos.

- En cuanto a las razones para no presentarse a convocatorias internacionales para el financiamiento de proyectos, no residen en la falta de interés, sino en la falta de apoyo institucional por parte de las organizaciones en las cuales se desempeñan. En síntesis: los tres primeros problemas identificados con idéntica jerarquía en ambos congresos son: "falta de información sobre las convocatorias", "falta de experiencia o capacitación para someter propuestas a las convocatorias" y "falta de tiempo".

En cuanto a los cambios registrados entre ambos congresos:

- La distancia tuvo un rol importante en el cambio en la composición de los asistentes ${ }^{11}$ a ambos congresos: en México se inscribió una mayoría de mexicanos $(47,9 \%)$ que en Argentina fueron minoritarios $(8,1 \%) \mathrm{y}$ algo similar sucedió en Buenos Aires (44,3\% y 14,8\%).

- La pirámide de edades de quienes respondieron la encuesta fue más equilibrada en Argentina, indicio muy positivo para el desarrollo y la renovación de la institución.

- Se produjo una reducción significativa en la proporción de encuestados con estudios superiores en el extranjero (pasó del 42,2\% al 33,5\%), indicio fuerte de la institucionalización de los estudios superiores en los países de la región.

- En cuanto a la lengua del país donde se realizan estudios superiores, el inglés y el portugués incrementaron su participación; el francés y el español la redujeron, pero continuaron siendo dominantes. ¿Esto habla de un desplazamiento del área de influencia de los estudios del trabajo desde Europa hacia América?

11 No confundir con quienes respondieron la encuesta. La información sobre los asistentes fue tomada de las fichas de inscripción. 
- La encuesta mexicana daba cuenta de una propensión a iniciar y culminar tardíamente los estudios de posgrado (obtención del doctorado después de los 50 años); la encuesta argentina es testigo de un fuerte adelantamiento de ese proceso (obtención a los 30 y a los 40 años), tendencia claramente relacionada con la institucionalización de los estudios del trabajo.

- Se registró un aumento significativo de la docencia en las categorías intermedias de dedicación, y de la gestión y la consultoría en las categorías de más baja dedicación. ¿Desplaza la docencia a la investigación en las universidades? ¿Se trata de un desarrollo incipiente del vínculo entre la investigación y la actividad productiva?

A partir de las nuevas preguntas incluidas en la encuesta realizada en Argentina:

- Sorprende la importancia sobresaliente asignada al factor de impacto entre los criterios para elegir el lugar donde publicar. ¿Se debe esto a una estrategia de posicionamiento de la producción o a una respuesta a demandas de las instituciones donde los investigadores están insertos (en su mayoría universidades)?

- Un 81,3\% de los encuestados considera que la calidad de las publicaciones es esencial o muy importante para la evaluación de su desempeño en las instituciones donde trabaja. Esto no significa que en dichas instituciones se tome en cuenta el factor de impacto de dichas publicaciones. Es posible, en cambio (como pensamos que sucede generalmente en las ciencias sociales), que se tenga sobre todo en cuenta la indización. Como no formulamos una pregunta que nos permita despejar esta incógnita, nos limitamos a tenerla en cuenta para insertarla en una futura encuesta.

Veamos ahora qué conclusión podemos extraer de esta serie de puntos de comparación respecto a la problemática de la profesionalización de los estudios del trabajo en América Latina.

En lo que respecta a las diferencias, son indicativas de la profundización del proceso de institucionalización reflejado, por ejemplo, en el mayor equilibrio de la pirámide de edades, en el adelantamiento de los estudios doctorales y en la reducción significativa de la proporción de encuestados con estudios superiores realizados en el extranjero.

En lo que respecta a las similitudes, a medida que avanzábamos en el análisis de los resultados de la encuesta aumentaba nuestra sorpresa por la escasa variación en las percepciones, no obstante el tiempo transcurrido y el cambio en la composición de los encuestados. A tal punto que, como adelantamos, nos parece posible esbozar una hipótesis sobre la existencia de una 
cultura institucional de los miembros de la Asociación. ¿Qué rasgos caracterizarían a dicha cultura?

Dada la reiteración de los rasgos, lo que diremos a continuación no es original respecto a las conclusiones que extrajimos de la primera encuesta en lo referente a la elección del tema por interés e inspiración personal, no por demanda de organismos internacionales, ministerios o sindicatos; a la conexión de lo primero (el modo de elección) con el valor atribuido a la cooperación con colegas extranjeros como fuente de nuevas ideas y metodologías y a la conexión de lo segundo (la fuente de financiación) con la no presentación a convocatorias internacionales para la obtención de recursos (reflejada en el fuerte predominio del financiamiento local), atribuida por los encuestados no a su desinterés sino a la falta - o la insuficiencia - de apoyo institucional (por parte de las universidades en las cuales se desenvolvía el $76 \%$ de los encuestados en ambos congresos).

\section{Referencias bibliográficas}

De Sierra, G.; M. A. Garretón; M. Murmis y H. Trindade (2007). Las ciencias sociales en América Latina en una mirada comparativa. En: H. Trindade, coord. Las ciencias sociales en América Latina en perspectiva comparada. México: Siglo XXI, pp. 17-52.

Gaillard, A.M.; J. Gaillard; J.M. Russell Barnard; S. Galina; A.A. Canesse; P. Pellegrini; V. Ugartemendia y P. Cardenas (2013). Drivers and outcomes of S\&T international collaboration activities: a case study of biologists from Argentina, Chile, Costa Rica, Mexico and Uruguay. En: J. Gaillard y R. Arvanitis, eds. Research collaborations between Europe and Latin America: mapping and understanding partnership. París: Éditions des Archives Contemporaines, pp. 157-191.

Gaillard, J.; V. Krishna y R. Waast (1997). Scientific communities in the developing world. Nueva Delhi: Sage Publications.

Murmis, M. (2007). Sociología, ciencia política, antropología: institucionalización, profesionalización e internacionalización en Argentina. En: H. Trindade, coord. Las ciencias sociales en América Latina en perspectiva comparada. México: Siglo XXI.

Thompson, G.; J. Frances; R. Levačić y J. Mitchell (1991). Markets, hierarchies and networks: the coordination of social life. Londres: Sage Publications.

Vaccarezza, L. (2000). Las estrategias de desempeño de la profesión académica: ciencia periférica y sustentabilidad del rol de investigador universitario. Redes, 7(15), pp. 15-43.

Van Looy, B.; M. Ranga; J. Callaert; K. Debackere y E. Zimmermann (2004). Combining entrepreneurial and scientific performance in academia: towards a compounded and reciprocal Matthew-effect? [en línea]. Research Policy, 33(3), pp. 425-441. DOI: 10.1016/j.respol.2003.09.004. 
Vinck, D. (2013). Formation des chercheurs et mobilité internationale: utilité pour le pays d'origine. En: O. Glassey; J.P. Leresche y O. Moeschler, dirs. Penser la valeur d'usage des sciences. París: Éditions des Archives Contemporaines, pp. 77-91.

Walter, J. y D. Szlechter (2012). La profesionalización de los estudios del trabajo en América Latina: resultados de una encuesta [en línea]. Revista Latino-americana de Estudos do Trabalho, 17(27), pp. 9-51. Disponible en: <http://relet.iesp.uerj.br/ Relet_27/Cap\%C3\%ADtulo\%2002\%20-\%20La\%20Profesionalizacion\%20de\%20 los\%20estudios\%20RELET\%2027\%20-\%20SE.pdf> [acceso 1/4/2018].

Walter, J. y D. Szlechter (2014a). Pioneros, institucionalización y profesionalización de los estudios del trabajo en América Latina. En: M. Kleiche-Dray y D. Villavicencio, coords. Cooperación, colaboración científica y movilidad internacional en América Latina. Buenos Aires: Consejo Latinoamericano de Ciencias Sociales, pp. 213-234.

Walter, J. y D. Szlechter (2014b). La Asociación Latinoamericana de Estudios del Trabajo y la profesionalización de las ciencias sociales del trabajo en América Latina. Estudios del Trabajo, 48, pp. 145-173. 
Anexo

\section{Cuestionario de la encuesta Los Estudios del Trabajo en América Latina}

Este cuestionario se inscribe en una investigación sobre la constitución del campo de los estudios del trabajo en América Latina. Su objetivo es indagar sobre la trayectoria formativa y profesional de los investigadores que se dedican a temáticas laborales. Las respuestas individuales al cuestionario son confidenciales. Los resultados de la investigación solo serán publicados en forma agregada.

Completar el cuestionario le insumirá aproximadamente quince minutos.

1. Desarrolla actividades docentes en: Grado $\square \quad$ Posgrado $\square \quad$ No realiza actividades docentes $\square$

2. ¿Ocupa algún cargo de responsabilidad institucional? Sí $\square \quad$ NO

Si responde positivamente, precise (para el cargo más importante):

La denominación del cargo: El nombre de la institución:

3.¿Desarrolla actividades de investigación? Sí $\square \quad$ NO $\square \quad$ ¿En la misma institución? Sí $\square \quad$ NO $\square$ Nombre de la institución donde realiza tareas de investigación (si es diferente de la institución de la cual es docente):

4. Tipo de institución donde realiza tareas de investigación (varias respuestas posibles]:

Universidad $\square \quad$ Centro de investigación público (no universitario) $\square$

Organismo público $\square \quad$ Centro de investigación de ONG $\square$

Organismo internacional $\square$ (precisar):

Otro:

5. Tipo de cargo que ocupa en la institución donde realiza tareas de investigación:

Cargo permanente / de tiempo completo

Contrato temporal / de tiempo completo (asistente, doctorando, posdoctorado, etc.) $\square$

De tiempo parcial $\square$ Consultor y/o investigador invitado

6. División de su tiempo de trabajo (en porcentaje aproximado):

Actividad docente: __ \% Investigación:__ $\%$ Gestión:__ $\%$

Consultoría: _ $\%$ Otro (especificar):

7. Precise su[s] área[s] de investigación:

Derecho $\square$ Economía $\square$ Sociología $\square$ Gestión/management $\square$ Ergonomía

Comunicación / periodismo $\square \quad$ Otras ciencias sociales o humanas $\square$ Otra (especificar):

8. ¿Hizo sus estudios superiores (o parte de sus estudios) en el extranjero? Sí $\square \quad$ NO $\square$ Si la respuesta es Sí, por favor mencionar el[los] país[países]:

9. ¿Realizó estadías de investigación en el extranjero después de concluir sus estudios?:

sí $\square$ NO

Si la respuesta es Sí, indique la opción correcta consignando el o los países donde realizó las estadías:

Sabático: Estadía pagada por su institución:

Empleado por una institución extranjera internacional:

Estadía realizada por razones no profesionales:

10. ¿Ha sometido proyectos a convocatorias internacionales? [call for offers, call for projects]:

sí $\square$ NO

11. Si la respuesta es Sí, indique el origen de la convocatoria (varias respuestas posibles]:

Unión Europea o un país europeo (especificar):

EE.UU. $\square$ Canadá

Una fundación de investigación (Friedrich Ebert, Ford, etc., precisar su nombre):

ОІт $\square$ Un organismo internacional (especificar): Otros (especificar): 
12. Si no se presentó a ninguna convocatoria internacional, ¿cuáles fueron los motivos? (marque con $X$ en función de la importancia que se la da a cada motivo]:

\begin{tabular}{|c|c|c|c|c|c|}
\hline & $\begin{array}{l}\text { Sin } \\
\text { importancia }\end{array}$ & $\begin{array}{l}\text { Poco } \\
\text { importante }\end{array}$ & Importante & $\begin{array}{l}\text { Muy } \\
\text { importante }\end{array}$ & $\begin{array}{l}\text { Obstáculo } \\
\text { prohibitivo }\end{array}$ \\
\hline $\begin{array}{l}\text { Falta de información sobre las } \\
\text { convocatorias }\end{array}$ & $\square$ & $\square$ & $\square$ & $\square$ & $\square$ \\
\hline $\begin{array}{l}\text { Dificultad para conseguir contrapartes o } \\
\text { crear consorcios }\end{array}$ & $\square$ & $\square$ & $\square$ & $\square$ & $\square$ \\
\hline $\begin{array}{l}\text { Falta de experiencia o capacitación para } \\
\text { someter propuestas a las convocatorias }\end{array}$ & $\square$ & $\square$ & $\square$ & $\square$ & $\square$ \\
\hline $\begin{array}{l}\text { Falta de un marco legal o jurídico nacional } \\
\text { que permita participar }\end{array}$ & $\square$ & $\square$ & $\square$ & $\square$ & $\square$ \\
\hline $\begin{array}{l}\text { Dificultades vinculadas a las normativas } \\
\text { financieras y contables de su institución }\end{array}$ & $\square$ & $\square$ & $\square$ & $\square$ & $\square$ \\
\hline Problemas de coordinación & $\square$ & $\square$ & $\square$ & $\square$ & $\square$ \\
\hline Problemas culturales y de idioma & $\square$ & $\square$ & $\square$ & $\square$ & $\square$ \\
\hline $\begin{array}{l}\text { Esfuerzo demasiado importante en } \\
\text { relación con el monto que se obtiene }\end{array}$ & $\square$ & $\square$ & $\square$ & $\square$ & $\square$ \\
\hline No necesita este apoyo & $\square$ & $\square$ & $\square$ & $\square$ & $\square$ \\
\hline $\begin{array}{l}\text { Su institución no tiene el nivel científico } \\
\text { suficiente }\end{array}$ & $\square$ & $\square$ & $\square$ & $\square$ & $\square$ \\
\hline $\begin{array}{l}\text { Demasiada selectividad de las } \\
\text { convocatorias }\end{array}$ & $\square$ & $\square$ & $\square$ & $\square$ & $\square$ \\
\hline Falta de tiempo & $\square$ & $\square$ & $\square$ & $\square$ & $\square$ \\
\hline Otro (especificar): & $\square$ & $\square$ & $\square$ & $\square$ & $\square$ \\
\hline
\end{tabular}

13. Resultados o consecuencias de su colaboración con colegas extranjeros (marque con $X$ en función de la importancia que se le da a cada resultado]:

\begin{tabular}{|c|c|c|c|c|c|}
\hline & Insignificante & $\begin{array}{c}\text { Poco } \\
\text { importante }\end{array}$ & Importante & $\begin{array}{l}\text { Muy } \\
\text { importante }\end{array}$ & Esencial \\
\hline $\begin{array}{l}\text { Desarrollo de nuevas metodologías } \\
\text { de investigación }\end{array}$ & $\square$ & $\square$ & $\square$ & $\square$ & $\square$ \\
\hline $\begin{array}{l}\text { Desarrollo de una nueva temática } \\
\text { de investigación }\end{array}$ & $\square$ & $\square$ & $\square$ & $\square$ & $\square$ \\
\hline Reconocimiento en la propia institución & $\square$ & $\square$ & $\square$ & $\square$ & $\square$ \\
\hline $\begin{array}{l}\text { Reconocimiento científico a nivel } \\
\text { internacional }\end{array}$ & $\square$ & $\square$ & $\square$ & $\square$ & $\square$ \\
\hline Publicación en revistas extranjeras & $\square$ & $\square$ & $\square$ & $\square$ & $\square$ \\
\hline $\begin{array}{l}\text { Aumento del financiamiento para } \\
\text { su institución de pertenencia }\end{array}$ & $\square$ & $\square$ & $\square$ & $\square$ & $\square$ \\
\hline $\begin{array}{l}\text { Refuerzo de los vínculos con } \\
\text { contrapartes internacionales }\end{array}$ & $\square$ & $\square$ & $\square$ & $\square$ & $\square$ \\
\hline $\begin{array}{l}\text { Participación en nuevos proyectos } \\
\text { científicos }\end{array}$ & $\square$ & $\square$ & $\square$ & $\square$ & $\square$ \\
\hline
\end{tabular}




\begin{tabular}{llllll}
\hline $\begin{array}{l}\text { Participación en congresos, formaciones, } \\
\text { etcétera. }\end{array}$ & $\square$ & $\square$ & $\square$ & $\square$ & $\square$ \\
\hline $\begin{array}{l}\text { Acceso a datos e información no } \\
\text { disponibles en su país }\end{array}$ & $\square$ & $\square$ & $\square$ & $\square$ & $\square$ \\
\hline Otro (especificar): & $\square$ & $\square$ & $\square$ & $\square$ & $\square$ \\
\hline
\end{tabular}

14. ¿Cuál es su principal tema de investigación actual o de especialización profesional?:

¿Desde cuándo trabaja ese tema?: Es nuevo $\square \quad 1$ a 2 años $\square \quad 3$ a 5 años $\square$ más de 5 años

15. Mencione entre las siguientes posibles razones para elegir este tema de investigación aquellas que le parece que se aproximarían más a su propia elección (marque con $X$ en función de la importancia que se le da a cada razón ]:

\begin{tabular}{lccccc}
\hline & Insignificante & $\begin{array}{c}\text { Poco } \\
\text { importante }\end{array}$ & Importante & $\begin{array}{c}\text { Muy } \\
\text { importante }\end{array}$ & Esencial \\
\hline $\begin{array}{l}\text { Interés personal y/o curiosidad } \\
\text { intelectual }\end{array}$ & $\square$ & $\square$ & $\square$ & $\square$ & $\square$ \\
\hline $\begin{array}{l}\text { Importancia social y política del tema } \\
\text { para América Latina }\end{array}$ & $\square$ & $\square$ & $\square$ & $\square$ & $\square$ \\
\hline $\begin{array}{l}\text { Permite publicar en revistas y libros } \\
\text { a nivel internacional }\end{array}$ & $\square$ & $\square$ & $\square$ & $\square$ & $\square$ \\
\hline $\begin{array}{l}\text { Respuesta a un pedido de organismos } \\
\text { nacionales o internacionales } \\
\text { (PNuD, olt, unESco, IDRC, etcétera) }\end{array}$ & $\square$ & $\square$ & $\square$ & $\square$ & $\square$ \\
\hline $\begin{array}{l}\text { Respuesta a un pedido de sindicatos, } \\
\text { ministerios u otros organismos } \\
\text { nacionales }\end{array}$ & $\square$ & $\square$ & $\square$ & $\square$ & $\square$ \\
\hline $\begin{array}{l}\text { Tema novedoso al que se puede } \\
\text { contribuir de manera original }\end{array}$ & $\square$ & $\square$ & $\square$ & $\square$ & $\square$ \\
\hline $\begin{array}{l}\text { Tema de su investigación para } \\
\text { la maestría o el doctorado }\end{array}$ & $\square$ & $\square$ & $\square$ & $\square$ & $\square$ \\
\hline $\begin{array}{l}\text { Tema de investigación de las redes } \\
\text { científicas de pertenencia }\end{array}$ & $\square$ & $\square$ & $\square$ & $\square$ & $\square$ \\
\hline $\begin{array}{l}\text { Tema con amplia disponibilidad } \\
\text { de financiamiento }\end{array}$ & $\square$ & $\square$ & $\square$ & $\square$ & $\square$ \\
\hline $\begin{array}{l}\text { El tema corresponde a las orientaciones } \\
\text { de su equipo de investigación }\end{array}$ & $\square$ & $\square$ & $\square$ & $\square$ & $\square$ \\
\hline $\begin{array}{l}\text { Tema vinculado con actividades } \\
\text { docentes }\end{array}$ & $\square$ & $\square$ & $\square$ & $\square$ & $\square$ \\
\hline \begin{tabular}{l} 
Otras (mencionar): \\
\hline
\end{tabular} & $\square$ & $\square$ & $\square$ & $\square$ & $\square$ \\
\hline
\end{tabular}

16. Pertenencia a asociaciones nacionales o internacionales vinculadas con su campo de ejercicio profesional (indique el nombre o las siglas): 
17. Participación en congresos nacionales e internacionales durante los últimos dos años (indicar las siglas del congreso o la entidad organizadora):

\begin{tabular}{lll}
\hline & Congresos nacionales & Congresos internacionales \\
\hline Estudios del trabajo & \\
\hline Relaciones laborales & \\
\hline Otros (especificar): & \\
\hline
\end{tabular}

18. ¿Participó en la creación de revistas sobre temas laborales 0 afines? [aportar datos aproximados de las revistas que nos permitan identificarlas]:

19. Revistas de toda índole cuyo comité editorial, científico o evaluador ha integrado, incluso fuera del campo de los estudios laborales (mencione las tres principales y especifique el tipo de comité que integró]: a.

b. _ c.

20. ¿Ha publicado en revistas de estudios laborales [consigne los nombres aproximados de las revistas]?: En español:

En portugués: En francés:

En inglés: Otras:

21. ¿Ha publicado en revistas no relacionadas directamente con temáticas laborales?:

a. De la institución a la que pertenece $\square$

b. De una asociación de estudios del trabajo (ámbito latinoamericano)

c. Otras revistas académicas extranjeras:

En español o portugués $\square \quad$ En francés o inglés $\square \quad$ En otras lenguas (precisar):

Otras revistas:

22. A la hora de elegir una revista en la cual publicar: ¿qué orden de importancia le asigna a los siguientes ocho factores? (ordénelos de 1 a 8 , siendo 1 el más importante):

Referato ciego de pares que promete la revista ( )

Reputación que tiene la revista dentro de su ámbito académico / profesional ( )

Presencia de la revista en índices (Web of Science, Scopus, Scielo ...) ( )

Conocer a personas que dirigen la revista o forman parte del comité editorial ( )

Invitaciones que le han hecho para publicar en ella (dossier, números especiales, invitaciones] [ ]

Revista publicada por su institución ( )

Revista en español ( )

Factor de impacto de la revista ( )

23. ¿Ha publicado en revistas que requieren un pago para la publicación?: Sí $\square$ NO $\square$

24. ¿Ha actuado como consultor de organismos nacionales o internacionales? (mencione los organismos]:

25. Sus proyectos de investigación actuales son:

a. Proyectos individuales $\square$

b. Proyectos colectivos $\square$ - con financiamiento nacional

- con financiamiento internacional $\square$

26. Temática del (o los] proyecto: 
27. Fuentes de financiamiento de sus proyectos en los últimos cinco años (más de una opción posible): Nacional $\square \quad$ EE.UU $\square \quad$ Europa $\square$ Organismo internacional (OIT, PNUD, CLACSO, etc.) Otro (especificar):

28. ¿Aproximadamente, qué proporción del financiamiento de investigación que usted ha obtenido en los últimos cinco años tiene origen local o extranjero?: Local: __ Extranjero: _ $\%$

29. Si participó en proyectos con financiamiento internacional en los últimos cinco años ¿qué posición tuvo [o tiene] en dicho/s proyecto/s?: Coordinador general $\square$ Coordinador de un subequipo Investigador $\square$ Asistente $\square$ Otro (especificar: consultor externo, subcontratado, etc.):

30. Si participó en proyectos con financiamiento internacional en los últimos cinco años ¿cómo evalúa Ud. su contribución al proyecto que considera más importante?:

Indispensable para el desarrollo del proyecto $\square$ Importante para el desarrollo del proyecto $\square$

Papel limitado a algunas tareas $\square$ Participación puntual en el proyecto

31. ¿Cuál es su diploma de nivel más elevado?: Licenciatura $\square \quad$ Maestría $\square \quad$ Doctorado Otro (precisar):

Especialidad del diploma: Institución/país:

Tema y fecha de la tesis:

¿Prepara una tesis? (precisar nivel y tema):

32. ¿Ha participado en la creación de instituciones ligadas a la problemática del trabajo? Sí $\square$ NO

33. Si la respuesta es Sí, precise el tipo de institución:

\begin{tabular}{|c|c|c|c|c|c|c|}
\hline & \multicolumn{2}{|c|}{ Institución 1} & \multicolumn{2}{|c|}{ Institución 2} & \multicolumn{2}{|c|}{ Institución 3} \\
\hline \multicolumn{7}{|c|}{ Nombre y localización } \\
\hline \multicolumn{7}{|c|}{ Cargo que tuvo o tiene en ella } \\
\hline \multirow{4}{*}{ Tipo de institución } & Pública & $\square$ & Pública & $\square$ & Pública & $\square$ \\
\hline & Privada & $\square$ & Privada & $\square$ & Privada & $\square$ \\
\hline & Asociativa & $\square$ & Asociativa & $\square$ & Asociativa & $\square$ \\
\hline & Otra & $\square$ & Otra & $\square$ & Otra & $\square$ \\
\hline
\end{tabular}

34. Datos personales: Aclaración: gracias por indicar sus datos personales para que podamos enviarle los resultados de la encuesta. Solicitamos su año de nacimiento porque intentaremos identificar generaciones entre los especialistas en estudios del trabajo en América latina. También preguntamos por el país de origen y residencia a los efectos de estudiar las relaciones de cooperación internacional: Nombre y apellido:

Correo electrónico:

Año de nacimiento:

País y localidad de nacimiento:

País y localidad de residencia actual:

35. ¿Participó anteriormente en Congresos de ALAST? NO $\square$ Sí $\square \quad$ ¿Cuántos?

36. ¿Continuará participando en Congresos de ALAST después de este? NO $\square$ Sí

36. Pregunta abierta: Si lo desea, formule un breve comentario sobre la situación actual de los estudios del trabajo que considere útil comunicar a los directivos de ALAST

¡Gracias por su participación! Responsables de esta encuesta: Jorge Walter, Diego Szlechter, Rigas Arvanitis y Juan David Vanegas Daza. 